\title{
Clinical study of serum procalcitonin level in patients with myocardial infarction complicated by pulmonary infection
}

\author{
SHIMING SUN ${ }^{1}$, FENGLI WANG ${ }^{2}$, MIAO YU ${ }^{1}$ and JING KANG ${ }^{1}$ \\ ${ }^{1}$ Clinical Laboratory, The Third Affiliated Hospital of Qiqihar Medical University, Qiqihar, Heilongjiang 161000; \\ ${ }^{2}$ Clinical Laboratory, Dalian Municipal Central Hospital, Dalian, Liaoning 116033, P.R. China
}

Received March 28, 2018; Accepted September 27, 2018

DOI: 10.3892/etm.2018.6841

\begin{abstract}
This study determined the serum procalcitonin (PCT) levels in patients with myocardial infarction complicated by pulmonary infection and explore its clinical significance and diagnostic value. A total of 473 patients who were admitted to the Third Affiliated Hospital of Qiqihar Medical University from January 2016 to June 2017 were enrolled as research subjects. Patients were divided into four groups based on their symptom status in myocardial infarction and pulmonary infection. There were 109 patients in normal control group who did not experience symptoms of either myocardial infarction or pulmonary infection. Blood samples were collected from each patient, and PCT levels were measured. The data were analyzed. The serum PCT levels prior to treatment were compared with each other. The PCT levels in the myocardial infarction and the pulmonary infection group were all higher than that in the normal control group $(0.040 \pm 0.015)(\mathrm{p}<0.05)$. On the contrary, the serum PCT level in the myocardial infarction complicated by pulmonary infection group was higher than that in the normal control group $(p<0.001)$. The serum PCT level after treatment was compared with that before treatment within the same group. The serum PCT levels in the three disease groups were comparable after treatment. The differences in PCT levels before and after treatment were all statistically significant within all three groups $(\mathrm{p}<0.05)$. A patient's serum PCT level was correlated with myocardial infarction complicated by pulmonary infection, which suggested it can be used as an important diagnostic marker for this complication. This finding has important clinical value for predicting and evaluating the complicated condition of myocardial infarction and pulmonary infection by providing a more accurate, sensitive, and specific method for early diagnosis of the disease.
\end{abstract}

Correspondence to: Dr Shiming Sun, Clinical Laboratory, The Third Affiliated Hospital of Qiqihar Medical University, 27 Taishun Street, Qiqihar, Heilongjiang 161000, P.R. China

E-mail: mwc6nq@163.com

Key words: myocardial infarction, myocardial infarction complicated by pulmonary infection, serum procalcitonin

\section{Introduction}

Acute myocardial infarction (AMI) is one of the acute and critical cardiovascular diseases that are commonly seen in clinic. The patient often suffers from severe pain beneath the sternum, and the pain cannot be relieved with rest. Other symptoms include alteration of serum myocardial enzyme levels, abnormal electrocardiogram, abnormal hematological parameters, and increased body temperature $(1,2)$. AMI is an extremely dangerous condition with high mortality and disability rates. It seriously threatens patient's life and inflicts great pain to patients and their families (3). Clinically, myocardial infarction is often complicated by pulmonary infection. Myocardial infarction complicated by pulmonary infection poses a higher risk of death to patients and is more difficult to treat (4). Pulmonary infection is a common condition in clinic, which can be resulted from chemical, physical and allergic factors, and pathogenic microorganisms (5). Pulmonary infection requires timely and effective antibiotic intervention and other treatment measures. In recent years, basic medical research on this topic has continuously improved. A research report stated that serum levels of inflammatory factors were significantly elevated in patients with myocardial infarction complicated by pulmonary infection, which in turn promotes atherosclerosis, thereby creating a vicious circle. The complication increases difficulties in clinical treatments, and inflicts further harm to patients' health and life (6).

At present, the commonly used diagnostic indicators for evaluating inflammatory response in myocardial infarction include erythrocyte sedimentation rate (ESR), white blood cell (WBC) count, and high-sensitivity C-reactive protein (hs-CRP) (7-9). However, these diagnostic indicators have disadvantages and limitations such as strict requirement in sample collecting time, low sensitivity, poor specificity, easy contamination, antibiotic interference, high cost, and long waiting time, suggesting that they are not appropriate to be used for diagnosis of myocardial infarction complicated by pulmonary infection (10-12). Through comparative analysis, procalcitonin (PCT) was deemed to be not plagued by these disadvantageous factors. PCT is a sensitive and specific indicator of inflammation. So far it has not been reported in literature that serum PCT was ever used as a clinical diagnostic indicator of myocardial infarction complicated by pulmonary infection. In this study, the serum PCT level in patients with 
myocardial infarction complicated by pulmonary infection was determined, and its clinical significance and diagnostic value were explored.

\section{Materials and methods}

Materials. A total of 473 patients who were admitted to the Clinical Laboratory, the Third Affiliated Hospital of Qiqihar Medical University (Qiqihar, China) from January 2016 to June 2017 were enrolled as research subjects. Patients were divided into four groups based on their symptom status in myocardial infarction and pulmonary infection. There were 109 patients in normal control group who did not have either myocardial infarction or pulmonary infection; 118 patients in myocardial infarction group; 121 patients in pulmonary infection group; and 125 patients in myocardial infarction complicated by pulmonary infection group. The general clinical data such as age and sex were comparable, and there were no statistically significant differences between the four groups. Diagnosis of myocardial infarction was based on the 2012 ACC Guideline for the Management of ST-Elevation Myocardial Infarction. All subjects were inquired about their medical history, and underwent multiple examinations including physical, electrocardiography, ultrasonography, and coronary angiography. The diagnostic criteria for AMI recommended by the World Health Organization (WHO) were followed in the diagnosis. Specifically, the presence of two of the three indicators, i.e., chest pain, abnormal electrocardiogram and abnormal myocardial enzyme levels, was required to confirm the diagnosis of AMI. The diagnosis of pulmonary infection was based on the guidelines published by the Chinese Society of Respiratory Diseases in 2013. Basically, patients were diagnosed to have pulmonary infection by both pulmonary CT and sputum culture, and patients did not have a history of pulmonary infection in the past 1 year. Patients were diagnosed to have myocardial infarction complicated by pulmonary infection if all the diagnostic criteria mentioned above for both myocardial infarction and pulmonary infection were met at the same time.

Patients were excluded from the study if the following conditions were present: Cardiogenic shock, intoxication, peripheral vascular diseases, stroke, disturbance of consciousness, renal dysfunction, severe infection, sepsis, malignant tumors, acute pancreatitis, stable angina, thyroid diseases, autoimmune diseases, metabolic diseases, severe organ perfusion abnormalities, serious injury, severe liver dysfunction, severe burns, medullary thyroid $\mathrm{C}$ cell carcinoma, blood system diseases, cerebrovascular accident, and other diseases affecting PCT and inflammatory factors. Those patients who received anti-inflammatory drug treatment before admission were also excluded. This study was approved by the Medical Ethics Committee of the Third Affiliated Hospital of Qiqihar Medical University. All patients participating in this study or their families signed informed consent and volunteered to participate in this study.

Methods. Venous blood samples $(5 \mathrm{ml})$ were taken from all patients under fasting at following time-points: In the morning following admission; after pulmonary infection was diagnosed (at $48 \mathrm{~h}$ after admission for patients without pulmonary
Table I. General data of patients in the four groups.

\begin{tabular}{lcccc}
\hline & $\begin{array}{c}\text { Patient } \\
\text { no. }\end{array}$ & Male & Female & $\begin{array}{c}\text { Average } \\
\text { age (years) }\end{array}$ \\
\hline Normal control & 109 & 55 & 54 & $66.75 \pm 10.05$ \\
Myocardial infarction & 118 & 61 & 64 & $67.12 \pm 10.11$ \\
Pulmonary infection & 121 & 60 & 58 & $66.95 \pm 9.85$ \\
$\begin{array}{l}\text { Myocardial infarction } \\
\text { complicated by }\end{array}$ & 125 & 58 & 63 & $67.15 \pm 10.62$ \\
pulmonary infection & & & & \\
\hline
\end{tabular}

infection) and at discharge. The blood samples were centrifuged at $500 \mathrm{x} \mathrm{g}$ for $10 \mathrm{~min}$ at $4^{\circ} \mathrm{C}$ using a centrifuge manufactured by Anhui Zhongke Zhongjia Scientific Instrument Co., Ltd. (Hefei, China). The supernatant (serum) was collected and used for the following PCT analysis. The serum PCT level was determined using a PCT quantification kit in conjunction with the electrochemiluminescence immunoassay. The kit was used strictly in accordance with the manufacturer's protocol. The immunoassay was performed on an up-converting phosphor technology (UPT)-based immunoassay analyzer manufactured by Beijing Hotgen Biotechnology Co., Ltd. (Beijing, China). Up-converting phosphor (UCP) particles are used in the UPT immunoassay as the bio-label, which emit visible light in the wavelength range of 475-670 $\mathrm{nm}$ under excitation of infrared light with a wavelength $>780 \mathrm{~nm}$. The UPT immunoassay can allow for accurate determination of serum PCT levels. A PCT level $>0.5 \mathrm{ng} / \mathrm{ml}$ was used as a positive reference value.

Statistical analysis. The SPSS 21.0 (IBM Corp., Armonk, NY, USA) statistics software was used for statistical analysis. Measurement data that were normally distributed were expressed as mean \pm standard deviation. Measurement data that were not normally distributed were presented as median and interquartile range. The rank-sum test was used in measurement data comparison between groups. Qualitative data were expressed as a percentage (\%). The $\chi^{2}$ test was used in qualitative data comparison between groups. The significance level $\alpha$ was set as 0.05 .

\section{Results}

General data of patients in each group. As shown in Table I, the age and sex among patients in the four groups were comparable. There were no statistically significant differences between the groups $(\mathrm{p}>0.05)$.

Serum PCT levels before treatment. Before treatment, the serum PCT levels in the normal control, myocardial infarction, pulmonary infection, and myocardial infarction complicated by pulmonary infection group were $0.040 \pm 0.015,0.080 \pm 0.022$, $0.170 \pm 0.050$ and $0.580 \pm 0.075 \mathrm{ng} / \mathrm{ml}$, respectively (Table II). Compared with the normal control group, the PCT levels in other three groups were all higher, and the differences were statistically significant $(\mathrm{p}<0.05)$. It is worth pointing out that the PCT level in the myocardial infarction complicated 
Table II. Serum PCT levels before treatment.

\begin{tabular}{lcc}
\hline Groups & PCT level (ng/ml) & P-value \\
\hline Normal control & $0.040 \pm 0.015$ & \\
Myocardial infarction & $0.080 \pm 0.022$ & $<0.05$ \\
Pulmonary infection & $0.170 \pm 0.050$ & $<0.05$ \\
Myocardial infarction & $0.580 \pm 0.075$ & $<0.001$ \\
complicated by & & \\
pulmonary infection & & \\
\hline
\end{tabular}

PCT, procalcitonin.

Table III. Comparison of serum PCT levels before and after treatment.

\begin{tabular}{lccc}
\hline & \multicolumn{2}{c}{ PCT level $(\mathrm{ng} / \mathrm{ml})$} & \\
\cline { 2 - 3 } Groups & $\begin{array}{c}\text { Before } \\
\text { treatment }\end{array}$ & $\begin{array}{c}\text { After } \\
\text { treatment }\end{array}$ & P-value \\
\hline $\begin{array}{l}\text { Normal control } \\
\text { Myocardial infarction }\end{array}$ & $0.040 \pm 0.015$ & $0.039 \pm 0.019$ & $>0.05$ \\
$\begin{array}{l}\text { Pulmonary infection } \\
\text { Myocardial infarction } \\
\text { complicated by }\end{array}$ & $0.170 \pm 0.050$ & $0.041 \pm 0.021$ & $<0.05$ \\
pulmonary infection & & $0.042 \pm 0.020$ & $<0.05$ \\
\hline
\end{tabular}

PCT, procalcitonin.

by pulmonary infection group was higher than the positive reference value $(0.5 \mathrm{ng} / \mathrm{ml})$.

Comparison of serum PCT levels before and after treatment in four groups. The serum PCT levels in the four groups were comparable after treatment (Table III). Although the differences in PCT levels before and after treatment were all statistically significant within the three disease groups, the decrease in the myocardial infarction complicated by pulmonary infection group $(\mathrm{p}<0.001)$ was substantially larger than those in the myocardial infarction and the pulmonary infection group $(\mathrm{p}<0.05)$.

\section{Discussion}

AMI refers to myocardial necrosis caused by acute, severe and persistent ischemia or hypoxia when a coronary artery becomes occluded. Risk factors for a coronary artery occlusion include unhealthy lifestyles such as overeating, overwork, agitation, constipation and smoking(13). Myocardial ischemic necrosis is due to thrombotic occlusion of coronary arteries resulting from inflammatory mechanisms, or plaque rupture (14). Myocardial infarction has high morbidity and mortality with 5-year mortality being higher than that of malignant tumors. As a critical cardiovascular disease commonly seen in clinic, clinicians have always been paying a particular attention to AMI. In addition, research on AMI has become the focus of current research in cardiology. Although association of hs-CRP with AMI has been extensively studied, it does not reflect the inflammatory response induced by AMI due to its low specificity. In recent years, PCT has drawn much attention and gradually become a preferred indicator of inflammatory response induced by AMI. It has gained wide applications in the cardiovascular field (15).

PCT is an inactive precursor of calcitonin. First of all, preprocalcitonin (prePCT) is synthesized in the rough endoplasmic reticulum of thyroid parafollicular cells under normal physiological conditions. The prePCT then enters the endoplasmic reticulum membrane of other cells and undergoes glycosylation and specific enzymatic excision of $\mathrm{N}$-terminal signal peptide producing PCT (16). In recent years, significant increase in PCT level has been observed mainly in severe inflammatory reactions, multiple organ failure, and endotoxemia in the clinic. In other cases, such as mild infection, viral infection and autoimmune reaction, the PCT level generally remained the same or only slightly increased. According to literature, PCT is extensively used as a disease indicator in cardiovascular-related fields (17). Clinically, AMI results from a coronary artery occlusion leading to myocardial ischemic necrosis. This condition induces a sterile inflammatory response, in which PCT is an important indicator of the intensity (18). The serum PCT level and vascular endothelial growth factor-B (VEGF-B) expression level in elderly patients with AMI were reported to have certain relevance with the patients' prognosis. Related studies have demonstrated that quantitative PCT measurement has important guiding significance for diagnosis, assessment and prognosis of patients with myocardial infarction (19).

Infection is implicated in AMI. Hematological abnormality, increased body temperature and other inflammatory manifestations often occur in patients with AMI. The patients are prone to combined lung infection, which in turn is one of the leading causes of aggravating myocardial infarction. Early diagnosis of myocardial infarction complicated by pulmonary infection is a pressing issue that needs to be solved in clinical treatment and control of the condition (20). Those commonly used markers in the clinic, such as ESR and C-reactive protein are effective but not specific in diagnosis of myocardial infarction complicated by pulmonary infection. Lack of specific markers for accurate and rapid diagnosis and prediction of the complication prompted us to explore in this study if serum PCT can be used as a specific marker in diagnosis of the disease. The serum PCT level prior to treatment was compared with each group. The PCT levels $(\mathrm{ng} / \mathrm{ml})$ in the myocardial infarction group $(0.080 \pm 0.022)$ and in the pulmonary infection group $(0.170 \pm 0.050)$ were all higher than that in the normal control group $(0.040 \pm 0.015)$. Although the differences were statistically significant $(\mathrm{p}<0.05)$, these levels were all in the lower range $(<0.5 \mathrm{ng} / \mathrm{ml})$. On the contrary, the serum PCT level in the myocardial infarction complicated by pulmonary infection group $(0.580 \pm 0.075)$ was in a higher range $(>0.5 \mathrm{ng} / \mathrm{ml})$, and was substantially higher than that in the normal control group $(\mathrm{p}<0.001)$. The serum PCT level after treatment was compared with that before treatment within the same group. The serum PCT levels in the three disease groups were comparable after treatment, i.e., $0.042 \pm 0.020$ in the myocardial infarction group, $0.041 \pm 0.021$ in the pulmonary 
infection group, and $0.049 \pm 0.022$ in the myocardial infarction complicated by pulmonary infection group. Although the differences in PCT levels before and after treatment were all statistically significant within all three groups, the decrease in the myocardial infarction complicated by pulmonary infection group $(\mathrm{p}<0.001)$ was larger than those in the myocardial infarction group and the pulmonary infection group $(\mathrm{p}<0.05)$. These findings demonstrated that the serum PCT can be used as a specific diagnostic indicator of myocardial infarction complicated by pulmonary infection due to substantial increase in its level when the complication occurs.

When pulmonary infection occurs in patients with myocardial infarction, early and timely intervention of the infection has important clinical implications for improving the condition of myocardial infarction. Some patients may not show characteristic symptoms of pulmonary infection such as fever, abnormal results of complete blood count and chest X-ray in an early stage. Delayed diagnosis and treatment of patients with occult pulmonary infection may impact the treatment and recovery of myocardial infarction negatively. Therefore, rapid and accurate diagnosis of myocardial infarction complicated by pulmonary infection is crucial to patients' overall treatment and prognosis. In this study, it was found that the serum PCT level in patients with myocardial infarction complicated by pulmonary infection was significantly higher than that in patients with myocardial infarction only. Clinically, quantification of serum PCT in patients can help diagnose myocardial infarction complicated by pulmonary infection, which may play an important role in accurate diagnosis and timely treatment of the complication. In the present study, using a large number of subjects a validated threshold value of the serum PCT level in patients with myocardial infarction complicated by pulmonary infection was $0.5 \mathrm{ng} / \mathrm{ml}$, which was consistent with the literature (21). It was reported that the sensitivity of a serum PCT level $>0.5 \mathrm{ng} / \mathrm{ml}$ was $\sim 97 \%$ for the diagnosis of chronic cardiac insufficiency complicated by pulmonary infection $(22,23)$. The sensitivity and specificity of a threshold value of $2 \mathrm{ng} / \mathrm{ml}$ were 89 and 94\%, respectively (24,25). In the current study, the serum PCT level in patients with myocardial infarction complicated by pulmonary infection was $0.580 \mathrm{ng} / \mathrm{ml}$. This value had a sensitivity of $92.7 \%$, a specificity of $93 \%$ and a negative predictive value of $74.5 \%$. Both the sensitivity and the specificity were in a good range. Patients with myocardial infarction tend to have pulmonary infection, which in turn can worsen the condition of myocardial infarction. The serum PCT, as a new marker, has high specificity and high sensitivity when applied in the clinic, providing a strong support for diagnosis of myocardial infarction complicated by pulmonary infection.

In conclusion, the serum PCT level in patients with myocardial infarction complicated by pulmonary infection increased substantially above the positive reference value of $0.5 \mathrm{ng} / \mathrm{ml}$, and was significantly higher than that in the normal control, the myocardial infarction and the pulmonary infection group. After treatment, the serum PCT level in patients with myocardial infarction complicated by pulmonary infection decreased significantly to a level comparable to that in other groups. Serum PCT assay had a very high sensitivity and specificity for the diagnosis of myocardial infarction complicated by pulmonary infection. It helps in clinical diagnosis and prediction of myocardial infarction complicated by pulmonary infection, and allows patients to receive appropriate and effective treatment in the early stage.

\section{Acknowledgements}

Not applicable.

\section{Funding}

This study was supported by Qiqihar Science and Technology Project (SFZD-2016113).

\section{Availability of data and materials}

The datasets used and/or analyzed during the present study are available from the corresponding author on reasonable request.

\section{Authors' contributions}

SS drafted the manuscript and was responsible for treating the patients. FW collected the blood samples. MY and JK analyzed the serum PCT levels. All authors read and approved the final manuscript.

\section{Ethics approval and consent to participate}

The study was approved by the Ethics Committee of the Third Affiliated Hospital of Qiqihar Medical University (Qiqihar, China). Signed informed consents were obtained from the patients or the guardians.

\section{Patient consent for publication}

Not applicable.

\section{Competing interests}

The authors declare that they have no competing interests.

\section{References}

1. McCarthy CP, Januzzi JL Jr and Gaggin HK: Type 2 myocardial infarction - an evolving entity. Circ J 82: 309-315, 2018.

2. Osmak GJ, Titov BV, Matveeva NA, Bashinskaya VV, Shakhnovich RM, Sukhinina TS, Kukava NG, Ruda MY and Favorova OO: Impact of 9p21.3 region and atherosclerosis-related genes' variants on long-term recurrent hard cardiac events after a myocardial infarction. Gene 647: 283-288, 2018.

3. Hayıroğlu Mİ, Keskin M, Uzun AO, Türkkan C, Tekkeşin Aİ and Kozan O: What is the predictive value of ST segment depression in inferior leads in first acute anterior myocardial infarction? J Electrocardiol: Dec 12, 2017 (Epub ahead of print).

4. Hansen CP, Overvad K, Tetens I, Tjønneland A, Parner ET, Jakobsen MU and Dahm CC: Adherence to the Danish food-based dietary guidelines and risk of myocardial infarction: A cohort study. Public Health Nutr 1: 1-11, 2018

5. Kang I, Chang MY, Wight TN and Frevert CW: Proteoglycans as immunomodulators of the innate immune response to lung infection. J Histochem Cytochem 66: 241-259, 2018.

6. Vemula MH, Ganji R, Sivangala R, Jakkala K, Gaddam S, Penmetsa $S$ and Banerjee S: Mycobacterium tuberculosis zinc metalloprotease-1 elicits tuberculosis-specific humoral immune response independent of mycobacterial load in pulmonary and extra-pulmonary tuberculosis patients. Front Microbiol 7: 418, 2016. 
7. Fonseca FAH, Izar MC, Maugeri IML, Berwanger O, Damiani LP, Pinto IM, Szarf G, França CN, Bianco HT, Moreira FT, et al; BATTLE-AMI Investigators: Effects of four antiplatelet/statin combined strategies on immune and inflammatory responses in patients with acute myocardial infarction undergoing pharmacoinvasive strategy: Design and rationale of the $\mathrm{B}$ and $\mathrm{T}$ types of lymphocytes evaluation in acute myocardial infarction (BATTLE-AMI) study: Study protocol for a randomized controlled trial. Trials 18: 601, 2017.

8. Liang Y,Chen H and Wang P: Correlation of leukocyte and coronary lesion severity of acute myocardial infarction. Angiology: Nov 12, 2017 (Epub ahead of print). doi: 10.1177/0003319717740782.

9. TahtoE,JadricR,Pojskic Land KicicE: Neutrophil-to-lymphocyte ratio and its relation with markers of inflammation and myocardial necrosis in patients with acute coronary syndrome. Med Arh 71: 312-315, 2017.

10. Haasper C, Kalmbach M, Dikos GD, Meller R, Müller C, Krettek C, Hildebrand F and Frink M: Prognostic value of procalcitonin (PCT) and/or interleukin-6 (IL-6) plasma levels after multiple trauma for the development of multi organ dysfunction syndrome (MODS) or sepsis. Technol Health Care 18: 89-100, 2010.

11. Hu L, Shi Q, Shi M, Liu R and Wang C: Diagnostic value of PCT and CRP for detecting serious bacterial infections in patients with fever of unknown origin: A systematic review and meta-analysis. Appl Immunohistochem Mol Morphol 25: e61-e69, 2017.

12. He C, Wang B, Li D, Xu H and Shen Y: Performance of procalcitonin in diagnosing parapneumonic pleural effusions: A clinical study and meta-analysis. Medicine (Baltimore) 96: e7829, 2017.

13. Xu N, Tang XF, Yao Y, Zhao X, Chen J, Gao Z, Yang Y, Gao RL, $\mathrm{Xu}$ B and Yuan JQ: Predictive value of neutrophil to lymphocyte ratio in long-term outcomes of left main and/or three-vesse disease in patients with acute myocardial infarction. Catheter Cardiovasc Interv 91: 551-557, 2018.

14. Ambavane A, Lindahl B, Giannitsis E, Roiz J, Mendivil J, Frankenstein L, Body R, Christ M, Bingisser R, Alquezar A, et al; TRAPID-AMI investigators: Economic evaluation of the one-hour rule-out and rule-in algorithm for acute myocardial infarction using the high-sensitivity cardiac troponin $\mathrm{T}$ assay in the emergency department. PLoS One 12: e0187662, 2017.

15. Najafi A, Khodadadian A, Sanatkar M, Shariat Moharari R, Etezadi F, Ahmadi A, Imani F and Khajavi MR: The comparison of procalcitonin guidance administer antibiotics with empiric antibiotic therapy in critically ill patients admitted in intensive care unit. Acta Med Iran 53: 562-567, 2015.
16. Samsudin I and Vasikaran SD: Clinical utility and measurement of procalcitonin. Clin Biochem Rev 38: 59-68, 2017.

17. Seshadri P, Manoli K, Schneiderhan-Marra N, Anthes U, Wierzchowiec P, Bonrad K, Di Franco C and Torsi L: Low-picomolar, label-free procalcitonin analytical detection with an electrolyte-gated organic field-effect transistor based electronic immunosensor. Biosens Bioelectron 104: 113-119, 2018.

18. Karaca Y, Gündüz A, Türkmen S, Mentese A, Türedi S, Eryiğit U and Karahan SC: Diagnostic value of procalcitonin levels in acute mesenteric ischemia. Balkan Med J 32: 291-295, 2015.

19. Devaux Y, Vausort M, Azuaje F, Vaillant M, Lair ML, Gayat E, Lassus J, Ng LL, Kelly D, Wagner DR, et al: Low levels of vascular endothelial growth factor B predict left ventricular remodeling after acute myocardial infarction. J Card Fail 18: 330-337, 2012.

20. Blackburn RM, Zhao H, Pebody R, Hayward AC and Warren-Gash C: Laboratory-confirmed respiratory infections as predictors of hospital admission for myocardial infarction and stroke: Time-series analysis of English data for 2004-2015. Clin Infect Dis 67: 8-17, 2018

21. Kubková L, Spinar J, Pávková Goldbergová M, Jarkovský J and Pařenica J: Inflammatory response and C-reactive protein value in patient with acute coronary syndrome. Vnitr Lek 59: 981-988, 2013 (In Czech)

22. Drieux L, Brossier F, Sougakoff W and Jarlier V: Phenotypic detection of extended-spectrum beta-lactamase production in Enterobacteriaceae: Review and bench guide. Clin Microbiol Infect 14 (Suppl 1): 90-103, 2008.

23. Eliakim-Raz N, Yahav D, Paul M and Leibovici L: Duration of antibiotic treatment for acute pyelonephritis and septic urinary tract infection - 7 days or less versus longer treatment: Systematic review and meta-analysis of randomized controlled trials. J Antimicrob Chemother 68: 2183-2191, 2013.

24. Wang H, Cui N, Niu F, Xu H, Long Y and Liu D: Usefulness of procalcitonin for the diagnosis of infection in cardiac surgical patients. Zhonghua Wei Zhong Bing Ji Jiu Yi Xue 29: 897-901, 2017 (In Chinese).

25. Chakravarti SB, Reformina DA, Lee TM, Malhotra SP, Mosca RS and Bhatla P: Procalcitonin as a biomarker of bacterial infection in pediatric patients after congenital heart surgery. Ann Pediatr Cardiol 9: 115-119, 2016.

This work is licensed under a Creative Commons

Attribution-NonCommercial-NoDerivatives 4.0 International (CC BY-NC-ND 4.0) License. 